Gazi University
Journal of Science
http://dergipark.gov.tr/gujs

\title{
Ruled Surfaces and Tangent Bundle of Unit 2-Sphere of Natural Lift Curves
}

\author{
Emel KARACA ${ }^{1 *(D)}$, Mustafa CALISKAN ${ }^{2}$ (D) \\ ${ }^{1}$ Ankara Hacı Bayram Veli University, Department of Mathematics, Ankara, Turkey \\ ${ }^{2}$ Gazi University, Department of Mathematics, Ankara, Turkey
}

\begin{abstract}
Highlights
- A unique ruled surface is corresponded to the natural lift curve.

- Properties of ruled surfaces generated by natural lift curves are examined.

- A method is given for modelling motions on $T \bar{M}$ instead of $D S^{2}$.
\end{abstract}

\begin{tabular}{l} 
Article Info \\
\hline Received: 08/07/2019 \\
Accepted: 25/02/2020 \\
Keywords \\
\hline Natural lift \\
Tangent bundle \\
Unit dual sphere \\
Ruled surface \\
Study's map
\end{tabular}

\begin{abstract}
This article deals with the isomorphism between unit dual sphere, $D S^{2}$, and the subset of the tangent bundle of unit 2-sphere, $T \bar{M}$. According to E. Study mapping, a ruled surface in $\mathbb{R}^{3}$ corresponds to each curve on $D S^{2}$. Through this correspondence, a unique ruled surface in $\mathbb{R}^{3}$ is corresponded to natural lift curve on $T \bar{M}$. Then striction curve, shape operator, mean curvature and Gaussian curvature of these ruled surfaces obtained by the natural lift curves are calculated. Developabilitiy condition of these ruled surfaces is given. Finally, we give an example to support the main results.
\end{abstract}

\section{INTRODUCTION}

The theories of curves and surfaces have been applied to many research fields in differential geometry, kinematics, etc. In the literature, definition and properties of natural lift curve was first encountered in Thorpe's book, see [1]. The natural lift curve is defined as the curve constructed by the end points of the tangent vectors of the main curve. In $\mathbb{R}^{3}$, the properties of natural lift curve of a given curve were studied by Ergün and Çalışkan, see [2]. Frenet operators of the natural lift curve of a given curve were presented by using the angle between Darboux vector field and the binormal vector field of the main curve, see [3].

Ruled surfaces are used extensively in geometry, surface design, computer-aided geometric design, physics, etc. A ruled surface is defined as the surface represented by moving a straight line in $\mathbb{R}^{3}$, along a space curve defined as the base curve, see [4]. In the literature, there are a lot of studies about the properties of ruled surfaces, see [5-12].

W. K. Clifford pioneered the properties of dual numbers in 1873. Then E. Study constructed the correspondence between the geometry of lines in $\mathbb{R}^{3}$ and unit dual sphere, $D S^{2}$ by defining a mapping called E. Study mapping. This mapping says that the oriented straight lines in $\mathbb{R}^{3}$ are congruent with the points on $D S^{2}$, see [13]. A correspondence among $T S^{2}, D S^{2}$ and non-cylindirical ruled surfaces was given by Karakaş and Gündoğan, see [14]. In the light of this study, the correspondence between unit dual sphere and tangent bundle of unit 2-sphere, $T S^{2}$ was given by Bekar, Hathout and Yayl1, see [15]. In that study, the correspondence between an each curve on $T S^{2}$ and ruled surface in $\mathbb{R}^{3}$ was given. The striction curve and the results of developability condition of ruled surfaces were presented. Then the isomorphism between 
tangent bundle of pseudo-sphere and ruled surfaces in Minkowski space was given by the same authors, see [16]. In that article, the developability condition and involute-evolute curve couples on $T S_{1}^{2}$ and $T H^{2}$ were also presented.

However, there is no research about ruled surfaces obtained by the natural lift curves in $\mathbb{R}^{3}$ with the isomorphism between unit dual sphere and the subset of unit 2-sphere, $T \bar{M}$. The remainder of this paper is divided into four sections: Section 2 is about basic definitions and theorems about the topic. Section 3 covers the properties of dual vectors, unit dual sphere and the isomorphism among $T \bar{M}, D S^{2}$ and ruled surfaces obtained by the natural lift curves in $\mathbb{R}^{3}$. Section 4 deals with the developability condition for the ruled surfaces obtained by the natural lift curves. In the same section, we give an example to illustrate the main conclusion. Section 5 is about the conclusion. Furthermore, obtained results are discussed.

\section{PRELIMINARIES}

This section deals with concepts of natural lift curve of a given curve, the tangent bundle of unit 2-sphere of the natural lift curve, the theory for ruled surfaces, Weingarten map, mean curvature and Gaussian curvature of ruled surfaces obtained by the natural lift curves.

Definition 1. Assume that $S^{2}$ is the unit 2-sphere in $\mathbb{R}^{3}$. The tangent bundle of $S^{2}$ is

$$
T S^{2}=\left\{(\gamma, v) \in \mathbb{R}^{3} \times \mathbb{R}^{3}:|\gamma|=1,\langle\gamma, v\rangle=0\right\},
$$

where $|$,$| " and "〈, \rangle "$ denote the norm and inner product in $\mathbb{R}^{3}$, respectively, see [15].

Definition 2. Let $\Gamma: I \rightarrow \bar{M}$ be a smooth curve. Here $\bar{M}$ represents a hypersurface in $\mathbb{R}^{3} . \Gamma$ is defined as an integral curve of $X$

$$
\frac{d \Gamma(s)}{d s}=X(\Gamma(s))
$$

Here $X$ is taken as tangent vector field on $\bar{M}$, see [2]. Let $T \bar{M}$ be a subset of $T S^{2}$, defined by

$T \bar{M}=\left\{(\bar{\gamma}, \bar{v}) \in \mathbb{R}^{3} \times \mathbb{R}^{3}:|\bar{\gamma}|=1,\langle\bar{\gamma}, \bar{v}\rangle=0\right\}$.

Here $\bar{\gamma}$ and $\bar{v}$ are the derivatives of $\gamma$ and $v$, respectively.

Definition 3. For the curve $\Gamma, \bar{\Gamma}$ is defined as the natural lift of $\Gamma$ on $T \bar{M}$, which produces in the equation:

$$
\bar{\Gamma}(s)=(\bar{\gamma}(s), \bar{v}(s))=\left(\dot{\gamma}(\mathrm{s})_{\dot{\gamma}(\mathrm{s})}, \dot{v}(\mathrm{~s})_{\dot{v}(\mathrm{~s})}\right) .
$$

Accordingly, we can write

$$
\frac{d(\bar{\Gamma}(s))}{d s}=\frac{d}{d s}\left(\dot{\Gamma}(s)_{\Gamma(s)}\right)=D_{\dot{\Gamma}(s)} \dot{\Gamma}(s)
$$

Here $D$ refers the Levi-Civita connection in $\mathbb{R}^{3}$. We have

$T \bar{M}=\cup T_{p} \bar{M}, p \in \bar{M}$,

where $T_{p} \bar{M}$ is taken as the tangent space of $\bar{M}$ at $p$, see [2]. Here $\chi(\bar{M})$ is defined as the space of vector fields on the hypersurface $\bar{M}$. Assume that $\{T(s), N(s), B(s)\}$ and $\{\bar{T}(s), \bar{N}(s), \bar{B}(s)\}$ are Frenet frames of the curve $\Gamma(s)$ and the natural lift $\bar{\Gamma}(s)$, respectively. The following figure is about the representation of natural lift curve: 


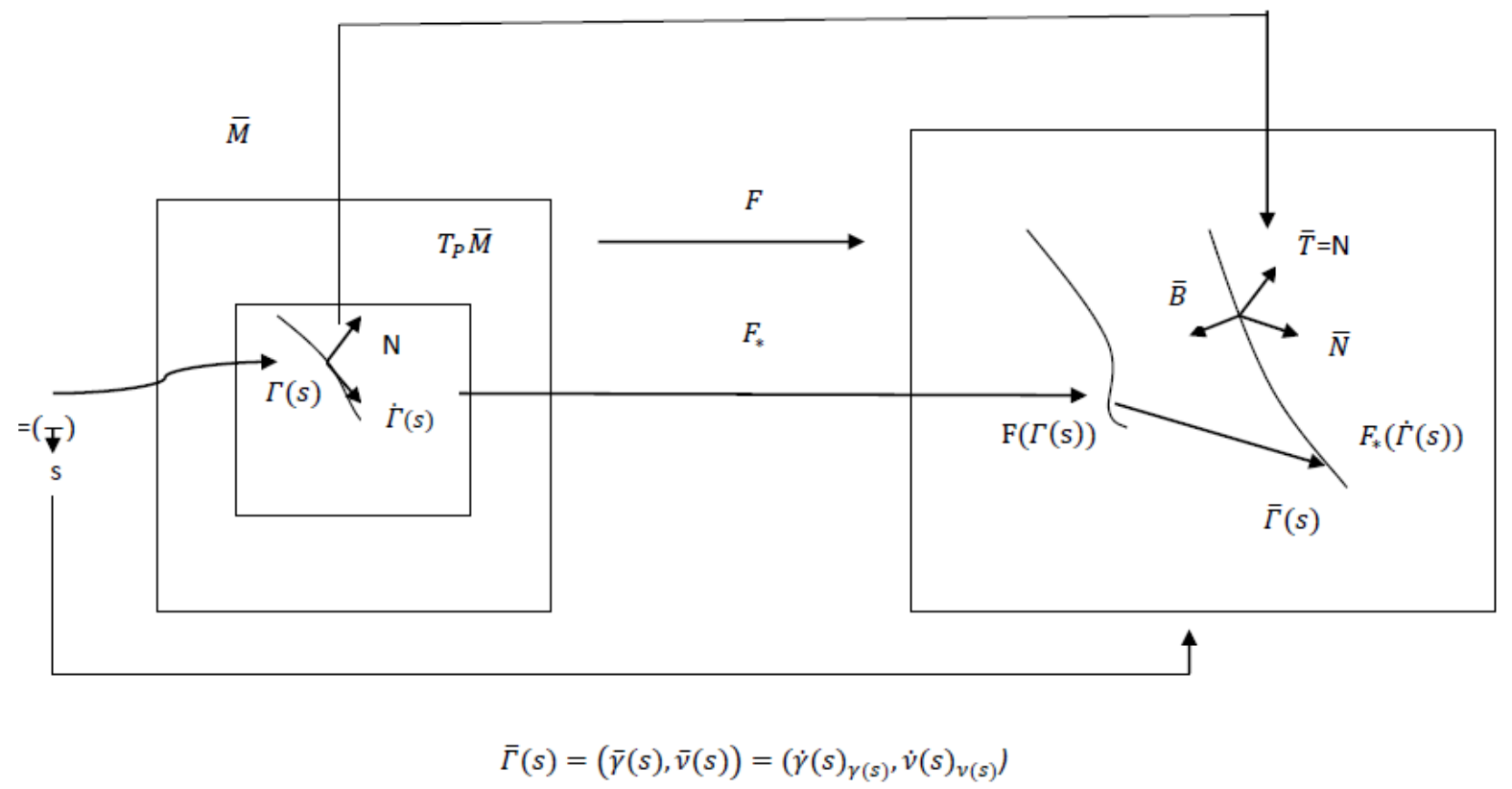

Figure 1. The natural lift curve of the main curve $\Gamma(s)$

In Figure 1,

$F: \bar{M} \rightarrow T \bar{M}$

and

$F_{* p}: T_{p} \bar{M} \rightarrow T_{F(p)}(T \bar{M})$

are transformations among $\bar{M}, T \bar{M}, T_{p} \bar{M}$ and $T_{F(p)}(T \bar{M})$.

For one-parameter family lines $\{\bar{\beta}(s), w(s)\}$, the ruled surface obtained by the set $\{\bar{\beta}(s), w(s)\}$ is

$\bar{\phi}(s, v)=\bar{\beta}(s)+v \cdot w(s), s \in I, v \in \mathbb{R}$.

The striction curve is defined as:

$\check{\beta}(s)=\bar{\beta}(s) \frac{\langle\dot{\bar{\beta}}(s), \dot{w}(s)\rangle}{\langle\dot{w}(s), \dot{w}(s)\rangle} \cdot w(s)$.

The striction curve $\breve{\beta}(s)$ is congruent with the base curve $\bar{\beta}(s)$ if the multiplication $\dot{\bar{\beta}}(s)$ and $\dot{w}(s)$ is equal to zero.

Definition 4. Let $\bar{M}$ be a hypersurface in $\mathbb{R}^{3}$. Nis the unit normal vector of $\bar{M}$. For every $\chi \in \chi(\bar{M})$,

$S: \chi(\bar{M}) \rightarrow \chi(\bar{M})$,

$X \mapsto S(X)=D_{X} N$

is called Weingarten map (shape operator) on $\bar{M}$. Here $D$ is Riemannian connection in $\mathbb{R}^{3}$, see [4].

Definition 5. Assume that $N$ is unit orthogonal vector field on $\bar{M}$.

$S_{p}: T_{p} \bar{M} \rightarrow T_{p} \bar{M}$ 
$X \mapsto S(X)=D_{X} N$

is called Weingarten map at for each point $p$ on $\bar{M}$, see [4]. The unit normal vector of $\bar{M}$ is defined as

$N=\frac{\partial_{1} \times \partial_{2}}{\left\|\partial_{1} \times \partial_{2}\right\|}$

Definition 6. Let $\bar{M}$ be a hypersurface in $\mathbb{R}^{3}$.

$K: \bar{M} \rightarrow \mathbb{R}$,

$p \mapsto K(p)=\operatorname{det}(S(p))$

is called Gaussian curvature on $\bar{M}$. Here $S(p)$ is the shape operator at each point $p$, see [4].

Definition 7. Let $\bar{M}$ be a hypersurface in $\mathbb{R}^{3}$.

$H: \bar{M} \rightarrow \mathbb{R}$,

$p \mapsto H(p)=\frac{1}{2} \operatorname{Tr}(S(p))$

is called mean curvature on $\bar{M}$, see [4].

\section{RULED SURFACES OBTAINED BY NATURAL LIFT CURVES AND UNIT DUAL SPHERE}

This section deals with some properties and theorems about the dual vectors. Moreover, the correspondence between the unit dual sphere, $D S^{2}$, and the subset of the tangent bundle of unit 2-sphere, $T \bar{M}$ is given.

The set of dual numbers is given as

$I D=\left\{X=x+\varepsilon x^{*}:\left(x, x^{*}\right) \in \mathbb{R} \times \mathbb{R}, \varepsilon^{2}=0\right\}$.

Here $x$ and $x^{*}$ are real and dual parts of $X$. If $\vec{x}$ and $\vec{x}^{*}$ are vectors in $\mathbb{R}^{3}$, then $\vec{X}=\vec{x}+\varepsilon \vec{x}^{*}$ is called dual vector. Let $\vec{X}=\vec{x}+\varepsilon \vec{x}^{*}$ and $\vec{Y}=\vec{y}+\varepsilon \vec{y}^{*}$ be dual vectors. The addition, inner product and vector product are defined as follows:

the addition is given as:

$\vec{X}+\vec{Y}=(\vec{x}+\vec{y})+\varepsilon\left(\vec{x}^{*}+\vec{y}^{*}\right)$

and their inner product is

$\langle\vec{X}, \vec{Y}\rangle=\langle\vec{x}, \vec{y}\rangle+\varepsilon\left(\left\langle\vec{x}^{*}, \vec{y}\right\rangle+\left\langle\vec{x}, \vec{y}^{*}\right\rangle\right)$.

Also the vector product is given as

$\vec{X} \times \vec{Y}=(\vec{x} \times \vec{y})+\varepsilon\left(\vec{x}^{*} \times \vec{y}+\vec{x} \times \vec{y}^{*}\right)$,

where" $\times$ " is vector product in $\mathbb{R}^{3}$.

The norm of $\vec{X}=\vec{x}+\varepsilon \vec{x}^{*}$ is represented as:

$|\vec{X}|=\sqrt{\langle\vec{x}, \vec{x}\rangle}+\varepsilon \frac{\left\langle\vec{x}, \vec{x}^{*}\right\rangle}{\sqrt{\langle\vec{x}, \vec{x}\rangle}}$.

The norm of $\vec{X}$ exists only for $\vec{x} \neq 0$. If the norm of $\vec{X}$ is equal to 1 , the dual vector is called unit dual vector. Namely, $\langle\vec{x}, \vec{x}\rangle=1$ and $\left\langle\vec{x}, \vec{x}^{*}\right\rangle=0$ are satisfied. 
The unit dual sphere which consists of the all unit dual vectors is defined as:

$D S^{2}=\left\{\vec{X}=\vec{x}+\varepsilon \vec{x}^{*} \in I D^{3}:|\vec{X}|=1\right\}$.

For detailed information for dual vectors, see [1]. The correspondence between the unit dual sphere and the tangent bundle of unit 2-sphere of the natural lift curve is given via equation (1) and equation (10):

$T \bar{M} \rightarrow D S^{2}$,

$\bar{\Gamma}=(\bar{\gamma}, \bar{v}) \mapsto \vec{\Gamma}=\bar{\gamma}+\varepsilon \bar{v}$

Theorem 1. (E. Study mapping) There exists one-to-one congruence between the points of $D S^{2}$ and the oriented lines in $\mathbb{R}^{3}$, see [1].

Theorem 2. Assume that $\vec{\Gamma}(s)=\vec{\gamma}(s)+\varepsilon \overrightarrow{\vec{v}}(s)$ is a natural lift curve on $D S^{2}$ with parameter $s$. In $\mathbb{R}^{3}$, the ruled surface obtained by the natural lift curve $\bar{\Gamma}(\mathrm{s})$ can be represented as

$\bar{\phi}(s, \vartheta)=\bar{\gamma}(s) \times \bar{v}(s)+\vartheta \cdot \bar{\gamma}(s)$

where

$\hat{\beta}(s)=\bar{\gamma}(s) \times \bar{v}(s)$

is defined as the base curve of $\bar{\Phi}$, see [5].

Definition 8. Let $\bar{\Phi}$ be a ruled surface in $\mathbb{R}^{3}$. Assume that $E=\{X, Y\}$ is orthonormal base of $\chi(\bar{\phi})$, where $X$ is the tangent vector field of the rulling at the point $P \in M$. The matrix

$S_{\bar{\Phi}}=\left[\begin{array}{ll}<S(X), X> & <S(Y), X> \\ <S(X), Y> & <S(Y), Y>\end{array}\right]$

is called Weingarten map (shape operator) on $\bar{\Phi}$, see [12].

Furthermore, the standart unit normal vector field of $\bar{\Phi}$ is represented as:

$N_{1}=\frac{\bar{\phi}_{\mathrm{s}} \times \bar{\phi}_{\mathrm{v}}}{\left|\bar{\phi}_{\mathrm{s}} \times \bar{\phi}_{\mathrm{v}}\right|}$

The Gaussian curvature and mean curvature of $\bar{\Phi}$ are represented as:

$K=\frac{L N-M^{2}}{E G-F^{2}}$

and

$H=\frac{L G+E N-2 M F}{2\left(E G-F^{2}\right)}$

where $E=<\bar{\phi}_{\mathrm{s}}, \bar{\phi}_{\mathrm{s}}>, F=<\bar{\phi}_{\mathrm{s}}, \bar{\phi}_{\mathrm{v}}>$ and $G=<\bar{\phi}_{\mathrm{v}}, \bar{\phi}_{\mathrm{v}}>$, respectively. Also $L=\frac{\operatorname{det}\left(\bar{\phi}_{\mathrm{s}}, \bar{\phi}_{\mathrm{v}}, \bar{\phi}_{\mathrm{ss}}\right)}{\sqrt{E G-F^{2}}}, M=$ $\frac{\operatorname{det}\left(\bar{\phi}_{\mathrm{s}}, \bar{\phi}_{\mathrm{v}}, \bar{\phi}_{\mathrm{sv}}\right)}{\sqrt{E G-F^{2}}}$ and $N=\frac{\operatorname{det}\left(\bar{\phi}_{\mathrm{s}}, \bar{\phi}_{\mathrm{v}}, \bar{\phi}_{\mathrm{vv}}\right)}{\sqrt{E G-F^{2}}}$ are taken for calculations, see [11].

Moreover, the isomorphism among $T \bar{M}, D S^{2}$ and $\mathbb{R}^{3}$ can be given as:

$T \bar{M} \rightarrow D S^{2} \rightarrow \mathbb{R}^{3}$ 
$\bar{\Gamma}(s)=(\bar{\gamma}(s), \bar{v}(s)) \mapsto \bar{\Gamma}(s)=\bar{\gamma}(s)+\varepsilon \bar{v}(s) \mapsto \bar{\phi}(s, \vartheta)=\bar{\gamma}(s) \times \bar{v}(s)+\vartheta \cdot \bar{\gamma}(s)$

Using equation (12), we get the derivative of $\hat{\beta}(s)$ :

$\dot{\hat{\beta}}(s)=\dot{\vec{\gamma}}(s) \times \overrightarrow{\vec{v}}(s)+\overrightarrow{\vec{\gamma}}(s) \times \dot{\vec{v}}(s)=\dot{\vec{\gamma}}(s) \cdot \dot{\vec{v}}(s)=0$.

By taking $\bar{\gamma}(s)$ as unit, the developability condition is calculated as follows:

$\operatorname{det}(\dot{\hat{\beta}}(s), \overrightarrow{\vec{\gamma}}(s), \dot{\vec{\gamma}}(s))=(\dot{\vec{\gamma}}(s) \times \overrightarrow{\vec{v}}(s)+\overrightarrow{\vec{\gamma}}(s) \times \dot{\vec{v}}(s)) \cdot \dot{\vec{\gamma}}(s)$

$=\langle\dot{\vec{\gamma}}(s), \dot{\vec{v}}(s)\rangle=0$.

Corollary 1. Assume that $\vec{\Gamma}(s)=\vec{\gamma}(s)+\varepsilon \overrightarrow{\vec{v}}(s)$ is natural lift curve on $D S^{2}$.The ruled surface obtained by natural lift curve is developable if and only if

$\langle\dot{\vec{\gamma}}(s), \dot{\vec{v}}(s)\rangle=0$

The proof is a result of equation (13).

Proposition 1. Assume that $\vec{\Gamma}(s)=\overrightarrow{\vec{\gamma}}(s)+\varepsilon \overrightarrow{\vec{v}}(s)$ is natural lift curve of the curve on $D S^{2}$. The ruled surface obtained by the natural lift curve is developable if and only if $\vec{\Gamma}(s)$ and $\overrightarrow{\vec{\gamma}}(s)$ have the same arclength parameter.

Proof. Assume that $\vec{\Gamma}(s)=\overrightarrow{\vec{\gamma}}(s)+\varepsilon \overrightarrow{\vec{v}}(s)$ is natural lift curve on $D S^{2}$. The norm of $\vec{\Gamma}(s)$ is defined in the following equation:

$$
|\vec{\Gamma}(s)|=\sqrt{\langle\overrightarrow{\vec{\gamma}}(s), \overrightarrow{\vec{\gamma}}(s)\rangle}+\frac{\langle\overrightarrow{\vec{\gamma}}(s), \overrightarrow{\vec{v}}(s)\rangle}{\sqrt{\langle\overrightarrow{\vec{\gamma}}(s), \overrightarrow{\bar{\gamma}}(s)\rangle}}
$$

The arc-length parameter is

$\bar{s}=\int_{0}^{s}|\vec{\Gamma}(s)| d s=s+\varepsilon \int_{0}^{s} \frac{\overrightarrow{\vec{\gamma}}(s) \cdot \overrightarrow{\vec{v}}(s)}{\sqrt{\vec{\gamma}(s) \cdot \overrightarrow{\vec{\gamma}}(s)}}$.

Using Corollary 1, the developability condition is given as follows:

$\langle\dot{\vec{\gamma}}(s), \dot{\vec{v}}(s)\rangle=0$

Consequently, we obtain

$\bar{s}=s$.

Therefore, $\overrightarrow{\bar{\Gamma}}(s)$ and $\dot{\vec{\gamma}}(s)$ have the same arc-length parameter.

\section{TANGENT BUNDLE OF UNIT 2-SPHERE OF NATURAL LIFT CURVES AND RULED SURFACES}

This section deals with the developability condition for $\bar{\phi}$ in $\mathbb{R}^{3}$ obtained by a smooth curve $\bar{\Gamma}=(\bar{\gamma}, \bar{v}) \in$ $T \bar{M}$. 
Proposition 2. Assume that $\bar{\Gamma}=(\bar{\gamma}, \bar{v})$ is the natural lift curve of in $T \bar{M}$. The ruled surface obtained by the natural lift curve is developable if and only if

$\langle\dot{\vec{\gamma}}(s), \dot{\vec{v}}(s)\rangle=0$

The proof is a result of Corollary 1.

Proposition 3. Let $\bar{\Gamma}=(\bar{\gamma}, \bar{v})$ be a natural lift curve in $T \bar{M}$. The pair $(\bar{\gamma}, \bar{v})$ is an involute-evolute curve pairs iff the ruled surface obtained by the curve $\bar{\Gamma}$ is developable.

Proposition 4. Assume that $\bar{\Gamma}=(\bar{\gamma}, \bar{v})$ be a natural lift curve in $T \bar{M}$. Provided that the pair $(\bar{\beta}, \bar{\gamma})$ is an involute-evolute curve pairs, the striction curve $\bar{\beta}$ and the base curve $\bar{\beta}(s)$ of $\bar{\Phi}(s, v)$ obtained by the natural lift curve are congruent.

Example 1. Let us take $\bar{\gamma}(s)=(-\sin s, \cos s, 0)$ on $\bar{M}$ and the vector $\bar{v}(s)=(\cos s, \sin s, 5)$ in $\mathbb{R}^{3}$. Because $|\bar{\gamma}(s)|=1$ and $\langle\bar{\gamma}, \bar{v}\rangle=0$, the natural lift $\bar{\Gamma}=(\bar{\gamma}, \bar{v})$ is in $T \bar{M}$. The ruled surface obtained by the natural lift curve $\bar{\Gamma}$ in $\mathbb{R}^{3}$ is

$\bar{\phi}(s, v)=\bar{\gamma}(s) \times \bar{v}(s)+v \cdot \bar{\gamma}(s)=(5 \cos s-v \cdot \sin s,-5 \sin s+v \cdot \cos s,-1)$.

Here the base curve is

$\hat{\beta}(s)=(5 \cos s, 5 \sin s,-1)$.

Moreover, we obtain $<\dot{\bar{\gamma}}(s), \dot{\bar{v}}(s)>=0$. Therefore, the ruled surface obtained by the curve $\bar{\Gamma}(s)$ is developable. The natural lift curve and the ruled surface generated by the natural lift curve are represented in Figure 2 as follows:
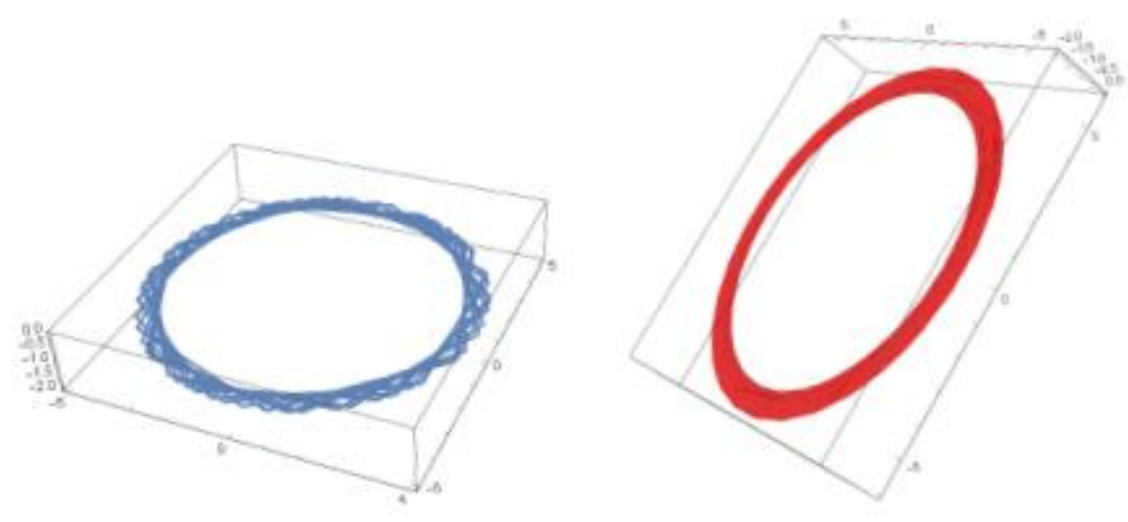

Figure 2. The natural lift curve and the ruled surface obtained by the natural lift curve $\bar{\Gamma}(s)$

Let us calculate the shape operator and Gaussian curvature of $\bar{\phi}$ :

$\bar{\phi}(s, v)=(5 \cos s-v \cdot \sin s,-5 \sin s+v \cdot \cos s,-1)$,

$\bar{\phi}_{s}(s, v)=(-5 \sin s-v \cdot \cos s, 5 \cos s-v \cdot \sin s, 0)$,

$\bar{\phi}_{v}(s, v)=(-\sin s, \cos s,-1)$.

Since $<\bar{\phi}_{s}, \bar{\phi}_{v}>=5 \neq 0$,the set $\left\{\bar{\phi}_{s}, \bar{\phi}_{v}\right\}$ is not orthogonal base. So, we will resolve these vectors to an orthonormal base byusing Gramm-Schmidt method. Then we calculate the matrix of shape operator with this orthonormal base.

For $\bar{\phi}_{s}$ and $\bar{\phi}_{v}$, the orthonormal base is 
$E_{1}=\frac{\bar{\phi}_{s}}{\left|\bar{\phi}_{s}\right|}=\frac{(5 \sin s-v \cdot \cos s, 5 \cos s-v \cdot \sin s, 0)}{\sqrt{25+v^{2}}}$,
$E_{2}=\frac{\bar{\phi}_{v}}{\left|\bar{\phi}_{v}\right|}=\frac{\left(5 \cdot v \cos s-v^{2} \cdot \sin s, 5 \cdot v \sin s+v^{2} \cdot \cos s,-v^{2}-25\right)}{\left(\sqrt{25+2 v^{2}}\right)\left(\sqrt{25+v^{2}}\right)}$.

From these equations, we obtain $\left\langle E_{1}, E_{2}\right\rangle=0$. The normal vector $N$ of the surface is

$N=E_{1} \times E_{2}$,

$N=\left(\frac{v \sin s-5 \cos s}{\sqrt{2 v^{2}+25}}, \frac{-5 \sin s-v \cos s}{\sqrt{2 v^{2}+25}}, \frac{v}{\sqrt{2 v^{2}+25}}\right)$.

Therefore, the shape operator is

$S\left(E_{1}\right)=\lambda_{1} \cdot E_{1}+\lambda_{2} \cdot E_{2}$,

$S\left(E_{2}\right)=\mu_{1} \cdot E_{1}+\mu_{2} \cdot E_{2}$,

where $\quad \lambda_{1}=\frac{-1}{\left|\bar{\phi}_{s}\right|^{3}\left|\bar{\phi}_{v}\right|} \operatorname{det}\left(\bar{\phi}_{s s}, \bar{\phi}_{s}, \bar{\phi}_{v}\right), \lambda_{2}=\mu_{1}=\frac{-1}{\left|\bar{\phi}_{s}\right|^{2}|| \bar{\phi}_{v \mid}{ }^{2}} \operatorname{det}\left(\bar{\phi}_{s v}, \bar{\phi}_{s}, \bar{\phi}_{v}\right), \quad \mu_{2}=\frac{-1}{\left|\bar{\Phi}_{s}\right| \cdot\left|\bar{\Phi}_{v}\right|^{3}} \operatorname{det}\left(\bar{\Phi}_{v v}, \bar{\Phi}_{s}, \bar{\Phi}_{v}\right)$ are taken for calculations of the coefficients for shape operator. After some calculations, we obtain

$S=\left(\begin{array}{cc}\frac{-1}{\sqrt{2} \cdot \sqrt{25+v^{2}}} & \frac{-5}{2 \cdot\left(25+v^{2}\right)} \\ \frac{-5}{2 \cdot\left(25+v^{2}\right)} & \frac{-\sqrt{25+v^{2}}}{2 \sqrt{2}}\end{array}\right)$.

The Gaussian curvature $K$ is

$K=\operatorname{det}(S)$

$K=\frac{v^{2}}{4 \cdot\left(25+v^{2}\right)}$.

The mean curvature $H$ is

$H=\frac{1}{2} \operatorname{tr}(S)$

$H=\frac{\left(-v^{2}+27\right)}{4 \sqrt{2} \cdot \sqrt{25+v^{2}}}$

\section{CONCLUSION}

E. Study mapping plays an important role to construct correspondence between Euclidean space and dual space. In this paper, the isomorphism between the subset of unit 2 -sphere, $T \bar{M}$, and unit dual sphere, $D S^{2}$ is constructed. Then we obtain ruled surfaces generated by the natural lift curves in $\mathbb{R}^{3}$. Using these results, it is possible to model motions by using the natural lift curves on the subset of unit 2-sphere instead of the unit dual sphere. Natural lift curves on $T \bar{M}$ can also be used to modeling motions in $\mathbb{R}^{3}$. 


\section{REFERENCES}

[1] Thorpe, J.A., "Elementary topics in differential geometry", Springer Verlag, New York, HeidelbergBerlin, (1979).

[2] Ergün, E., Çalışkan, M., “On natural lift of a curve”, Pure Mathematical Sciences, 2: 81-85, (2012).

[3] Ergün, E., Bilici, M., Çalışkan, M., "The Frenet vector fields and the curvatures of the natural lift curve", The Bulletin Society for Mathematical Services and Standarts, 2: 38-43, (2012).

[4] Do Carmo, M., "Differential geometry of curves and surfaces", Prentice-Hall, Inc., Englewood Cliffs, New Jersey, (1976).

[5] Yayl1, Y., Saraçoğlu, S., "Different approaches to ruled surfaces", SDU Journal of Science, 7: 56$68,(2012)$.

[6] Şentürk, G.Y., Yüce, S., "Characteristic properties of the ruled surface with Darboux frame in $\mathbb{R}^{3}$ ", Kuwait J Sci., 2: 14-33, (2015).

[7] Orbay, K., Aydemir, İ., "The ruled surfaces generated by Frenet vectors of a curve in $\mathbb{R}_{1}^{3}$ ", Cbu Journal of Science, 6: 155-160, (2010).

[8] Izumiya, S., Takeuchi, N., "Special curves and ruled surfaces", Contributions to Algebra and Geometry, 44: 203-212, (2003).

[9] Şenyurt, S., Çalışkan, A., "The quaternionic expression of ruled surfaces”, Filomat, 32: 5753-5766, (2018).

[10] Hacısalihoğlu, H.H., "On the pitch of a closed ruled surface", Mechanism and Machine Theory, 7: 291-305, (1972).

[11] Masal, M., Azak, A. Z., "The ruled surfaces according to type-2 Bishop frame in Euclidean 3-Space $E^{3}$," Mathematical Sciences and Applications E-Notes, 3: 74-83, (2015).

[12] Sarığlugil, A., Tutar, A., “On ruled surfaces in Euclidean space $E^{3}$ ”, Int. J. Contemp. Math. Sci.,2: 1-11, (2007).

[13] Fischer, I. S., "Dual-Number Methods in Kinematics and Dynamics", CRC Press, Boca Raton, London, New York, Washington DC: (1999).

[14] Karakaş, B., Gündoğan, H., "A relation among $D S^{2}, T S^{2}$ and non-cylindirical ruled surfaces", Mathematical Communications, 8: 9-14, (2003).

[15] Hathout, F., Bekar, M., Yayl1, Y., "Ruled surfaces and tangent bundle of unit 2-sphere", International Journal of Geometric Methods in Modern Physics, 2: (2017).

[16] Bekar, M., Hathout, F., Yayl1, Y., "Tangent bundle of pseudo-sphere and ruled surfaces in Minkowski space", General Letters in Mathematics, 5: 58-70, (2018). 